\title{
THE EFFECT OF CHANGES IN THE PULMONARY VASCULAR BED PRODUCED BY ATROPINE, PULMONARY ENGORGEMENT, AND POSITIVE-PRESSURE BREATHING ON DIFFUSING AND MECHANICAL PROPERTIES OF THE LUNG*
}

\author{
By WALTER J. DALY, $\dagger$ JOSEPH C. ROSS, AND ROY H. BEHNKE
}

(From the Department of Medicine and the Heart Research Center, Indiana University School of Medicine, and Veterans Administration Hospital, Indianapolis, Ind.)

(Submitted for publication December 20, 1962; accepted March 7, 1963)

Several factors probably have some role in determining the volume of the effectively ventilated pulmonary capillary bed, including pulmonary blood flow, pulmonary vascular pressures, pulmonary blood volume, alveolar and blood gas tensions, and mechanical effects of ventilation.

Others have found that atropine sulfate, $2.0 \mathrm{mg}$ iv, increases cardiac output $(1,2)$, decreases central venous pressure $(1,2)$, and affects the distribution of the blood volume as shown by plethysmographic study of extremity venous volume (3). This drug, then, provides an unusual rearrangement of some of the determinants of pulmonary capillary function and, for the present study, was used either alone or in combination with positivepressure breathing or pulmonary vascular engorgement produced by G-suit inflation.

This study was carried out to provide further information concerning the interaction of cardiac output, pulmonary vascular pressure, and pulmonary blood volume as determinants of the volume of effectively ventilated capillaries by determining the effects of changes in the pulmonary vascular bed produced by atropine, pulmonary engorgement, and positive-pressure breathing on the diffusing and mechanical properties of the lung.

\footnotetext{
* This study was supported in part by research grants H-6228 and H-4080 from the National Heart Institute, in part by U. S. Air Force contract 33(616)8378, and in part by the Indiana Heart Association. This work was presented in part at the annual fall meeting of the American Physiological Society, Buffalo, N. Y., August, 1962, and in part at the midwestern section meeting of the American Federation for Clinical Research, Chicago, Ill., November, 1962.

$\dagger$ Part of this work was done during tenure of U. S. Public Health Service postdoctoral research fellowship HTS-5363.
}

\section{METHODS}

Subjects. These studies were carried out in a group of 17 trained, normal, male subjects with an age range from 22 to 34 and body surface area range from 1.85 to $2.20 \mathrm{~m}^{2}$. All studies were carried out no sooner than 3 hours after a light meal and after the subjects had rested at least 30 minutes in the laboratory.

Experimental conditions. Observations were made with the subject either supine or sitting, as required by the specific experimental procedure. Data obtained with the subject in different postures were collected on separate days and are not used for comparison. Observations were made before and beginning 10 minutes after the iv administration of $2.0 \mathrm{mg}$ of atropine sulfate.

Several subjects were studied before and during pressure-suit inflation over the lower part of the body, both before and after the administration of atropine sulfate. The suit used is a single-chamber, balloon-type garment ${ }^{1}$ that covers the feet, legs, and abdomen and can be inflated to the desired pressure within 5 seconds by a standard Air Force $G$ valve. The suit was laced on the subject carefully to provide even distribution of pressure. All determinations for comparison with those made during suit inflation were carried out with the subject wearing the laced but uninflated suit. All determinations made during suit inflation were carried out after the suit had been inflated to a pressure of $100 \mathrm{~mm} \mathrm{Hg}$ for $30 \mathrm{sec}-$ onds, a pressure which, in previous studies, produced a mean rise in central venous pressure of $25 \mathrm{~mm} \mathrm{Hg}$ in seated subjects (4) and increases of $1.35 \mathrm{~mm} \mathrm{Hg}$ in intrathoracic pressure, $5 \mathrm{~mm} \mathrm{Hg}$ in gastric pressure, and $18 \mathrm{~mm} \mathrm{Hg}$ in rectal pressure in supine subjects (5). Subjects were trained to keep the glottis open during suit inflation to avoid a Valsalva maneuver.

Positive-pressure breathing was carried out from a closed system consisting of a 200-L drum, a Douglas valve, an underwater exhaust for flushing the system,

${ }^{1}$ This full pressure half-suit was made by the David Clark Co., Inc., Worcester, Mass., and in this paper, is referred to as a $G$ suit. This suit, however, is not a standard aviator's $G$ suit and cannot be used in that way, since it provides much more $G$ protection than the standard aviator $G$ suit, which comprises only abdominal, thigh, and skin bladders, but doesn't apply pressure to feet, ankles, knees, hips, and buttocks. 
and a $\mathrm{CO}_{2}$ absorber in the expiratory line (Figure 1). Because of the large volume of the system in relation to the tidal volume and because of a high air-flow rate through an underwater exhaust, respiratory pressure fluctuations in the system were minimal. Regulation of the rate of inflow of compressed air further regulated the pressure in the system. Pulmonary diffusing capacity was measured before and 2 minutes after beginning positive-pressure breathing.

Hemodynamic measurements. Cardiac output was determined in duplicate in the supine position by the indocyanine green dilution method with a Gilford IR 103 densitometer. Dye injections were made into the pulmonary artery during the end-expiratory phase of respiration. Cardiac output was calculated by the Hamilton semilog replotting method (6). Duplicate determinations of cardiac output by this method are reproducible with a mean difference of $3.8 \%$ in this laboratory. Duplicate determinations of cardiac output during maintained inflation of the $G$ suit or during positive-pressure breathing showed no greater variability if the dye injections were made during the period 30 seconds to $5 \mathrm{~min}$ utes after G-suit inflation or 1 to 3 minutes after beginning positive-pressure breathing. This suggests that within these time limits a reasonably steady state is attained so that the dye dilution method may be used to measure flow reliably.

Intravascular pressures were recorded by Statham P-23D transducers and a multichannel recording system. Pulmonary artery, right atrial, and brachial arterial pressures were recorded simultaneously, referred to the midthoracic level, electronically integrated, and recorded over several respiratory cycles.

Pulmonary blood volume estimations. Changes in pulmonary blood volume after atropine were estimated by the method described by Bondurant, Hickam, and Isley (7) and by Weissler, McCraw, and Warren (8). A Nuclear-Chicago 183-B scaling unit, 1810 radiation analyzer, and DS5-1 probe scintillation counter ${ }^{2}$ were used with the $20^{\circ}$ collimator. A convenient area in the right mid-lung field, well away from the mediastinal region and well above the diaphragm was selected for counting and marked on the skin. The collimator was applied directly to the skin in an axis perpendicular to the frontal plane of the body at a measured distance from the table top. After background counting over this area, $50 \mu \mathrm{c}$ of $\mathrm{I}^{131}$-albumin was injected intravenously. Twenty minutes later, counting was begun over the same region and then repeated 10 minutes after the iv administration of $2.0 \mathrm{mg}$ of atropine. In all cases, the counting rate was at least eight times the background rate. Three or more counting periods with a minimum of 1,680 counts each were used before and again after atropine. Between periods, the probe was removed from the skin, redirected, and replaced so that variability in probe placement and orientation could be made random. The variability among such periods was $2.4 \pm 1.5 \%$. Further extension of the time allowed for mixing of the

\footnotetext{
2 Nuclear-Chicago Corp., Des Plaines, Ill.
}

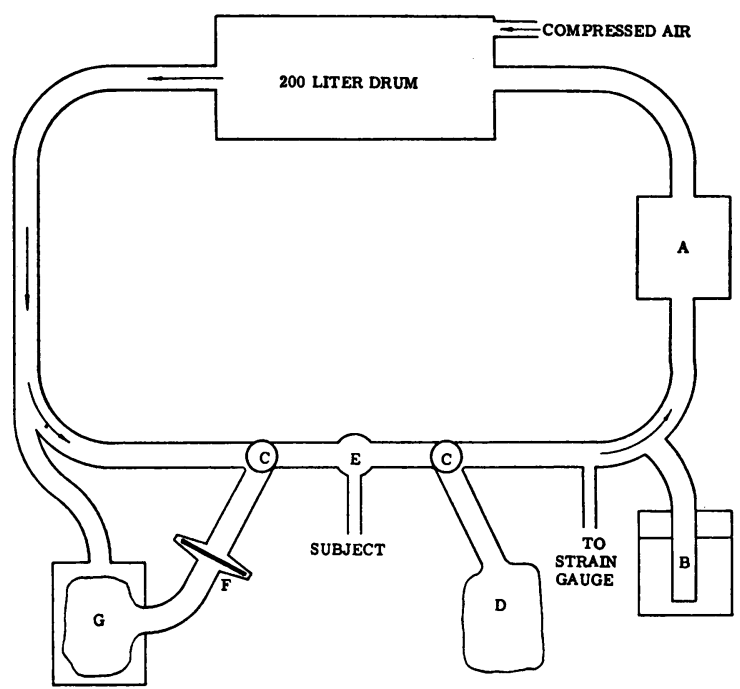

Fig. 1. Apparatus for POSITIVE-PRESSURe bREAThiNg. $\mathrm{A}=\mathrm{CO}_{2}$ absorber ; $\mathrm{B}=$ underwater exhaust (pressure in the system is determined by the depth of the exhaust tube under water; pressure fluctuations are minimized by keeping a high flow rate); $\mathrm{C}=$ three-way valves; $\mathrm{D}=\mathrm{bag}$ for collecting alveolar sample; $\mathrm{E}=$ Douglas valve; $\mathrm{F}=$ pneumotachograph; and $\mathrm{G}=$ gas bag containing $0.4 \% \mathrm{CO}$. Pressure is maintained by constant flow of compressed air, and recorded directly from a Statham P-23D strain gauge.

administered $\mathrm{I}^{131}$-albumin did not result in a change in the counting rate; consequently, altered counting rates observed after atropine do not represent further dilution of the administered dose. As was observed by Bondurant, Hickam, and Isley (7) and by Weissler, McCraw, and Warren (8), Valsalva maneuvers produced striking decreases in the counting rate over the lung. Percentage of change in pulmonary blood volume after atropine $=($ counts per minute after atropine - background counts per minute)/(counts per minute before atropine background counts per minute) $\times 100$.

Pulmonary diffusing capacity $\left(D_{L}\right)$ and capillary blood volume $\left(V_{c}\right)$ determinations. $D_{L}$ was determined by a modification of the Krogh breath-holding technique described by Forster and co-workers $(9,10)$ and as previously carried out in this laboratory (4). For calculation of $V_{c}$ by the method of Roughton and Forster (11), $D_{L}$ was determined at two different alveolar $\mathrm{O}_{2}$ tensions in each subject by using different concentrations of $\mathrm{O}_{2}$ in the inspired gas mixture. Determinations were made in duplicate under each condition. A period of at least 5 minutes was allowed between $D_{L}$ determinations. In the group in which the effects of atropine and G-suit inflation were studied, the gas mixture containing $0.4 \% \mathrm{CO}$ was inspired from a bag in a box connected to a spirometer, so that the inspired volume and breath-holding time were recorded on the spirometer tracing. When the determinations were made with ambient- and positive-pressure breathing before and after atropine, the area in the 
TABLE I

Lung volumes, airway resistance, and blood oxygen

\begin{tabular}{|c|c|c|c|c|c|c|c|c|c|c|c|c|}
\hline & \multicolumn{2}{|c|}{ FRC* } & \multicolumn{2}{|c|}{ Vs } & \multicolumn{2}{|c|}{ AR } & \multicolumn{2}{|c|}{$\mathrm{O}_{2} \mathrm{Cap}$} & \multicolumn{2}{|c|}{$\mathrm{O}_{2}$ Sat } & \multicolumn{2}{|c|}{$V_{D_{\text {(phys) }}}$} \\
\hline & B & A & B & A & B & A & B & A & B & A & B & $\bar{A}$ \\
\hline Mean & 3.46 & 3.32 & 0.61 & 0.54 & 1.82 & 1.45 & 19.2 & 19.4 & 96.0 & 96.0 & 139 & 166 \\
\hline $\mathrm{SD}$ & \multicolumn{2}{|c|}{$0.2^{0.00}$} & \multicolumn{2}{|c|}{0.2} & \multicolumn{2}{|c|}{0.05} & 0.7 & $4^{1.1}$ & 1.1 & 0.7 & \multicolumn{2}{|c|}{${ }_{0.01}^{13}$} \\
\hline
\end{tabular}

${ }^{*} \mathrm{FRC}=$ functional residual capacity, liters (BTPS, body temperature, pressure, saturated with water); Vs = slowly ventilated space, liters (BTPS); $A R=$ airway resistance, liters per second per centimeter $\mathrm{H}_{2} \mathrm{O} ; \mathrm{O}_{2}$ Cap = arterial blood oxygen capacity, volume per cent; $\mathrm{O}_{2}$ Sat $=$ oxyhemoglobin saturation, per cent; $\mathrm{VD}_{(\mathrm{phys})}=$ physiologic dead space, milliliters (BTPS); $\mathrm{B}=$ before atropine; and $\mathrm{A}=$ after atropine.

box around the bag containing the inspired mixture was not connected to a spirometer, but rather to the positivepressure breathing system already described (Figure 1), so that when measurements were made during pressure breathing, the gas mixture was inspired at the pressure at which the subject had been breathing. A pneumotachograph was interposed in the airway near the mouthpiece, and respiratory flow was integrated and recorded on a photographic recorder for determination of the inspired volume and breath-holding time. The apparatus dead space, which is included in the alveolar volume $\left(V_{A}\right)$, was small $(<100 \mathrm{ml})$ and constant under all conditions. By having the subject regulate the inspired volume, it was possible, in an individual subject, to determine $D_{L}$ at approximately the same $V_{A}$ under each condition studied.

$D_{L}$ was calculated with the Krogh equation (12) as modified by Forster and co-workers $(9,10): D_{L}(\mathrm{ml}$ $\mathrm{CO}_{\text {STPD }}$ per minute per mm Hg CO $)=\left[\left(V_{A}\right)(60)\right] /[(t)$ $\left.\left(P_{B}-47\right)\right] \times\left[\ln \left(F A_{C O_{o}} / F A_{C O}\right)\right] . \quad \mathrm{V}_{\mathrm{A}}$ is the alveolar volume (STPD, standard temperature, pressure, dry $-0^{\circ}$ $\mathrm{C}, 760 \mathrm{~mm} \mathrm{Hg}) ; t$ is the breath-holding time in seconds; $P_{B}-47$ is the barometric pressure less the vapor pressure of water at $37^{\circ} \mathrm{C}$ (in calculations of $D_{L}$ when subjects were breathing $10 \mathrm{~mm} \mathrm{Hg}$ positive pressure, this factor was $P_{B}+10-47 ; F A_{C O_{o}}$ is the initial alveolar $\mathrm{CO}$ concentration calculated by multiplying the inspired $\mathrm{CO}$ concentration by the ratio of the expired alveolar $\mathrm{He}$ concentration to inspired $\mathrm{He}$ concentration; and $\mathrm{FACO}_{t}$ is the alveolar $\mathrm{CO}$ concentration at the end of the breath-holding period as measured in the alveolar sample. A correction for capillary $\mathrm{CO}$ tension $\left(\mathrm{P}_{\mathrm{co}}\right)$ equilibrated with small amounts of $\mathrm{CO}$-hemoglobin present in the blood was made in each determination by subtracting the $\mathrm{P}_{\mathrm{co}}$ from both initial and final alveolar $\mathrm{CO}$ concentrations as previously described (9).

$V_{c}$ was calculated by the technique outlined in detail by Roughton and Forster (11). The mean capillary $\mathrm{O}_{2}$ tension was estimated by the technique suggested by McNeill, Rankin, and Forster (13). $\theta$ for the appropriate $\mathrm{O}_{2}$ tension was obtained from the data of Roughton, Forster, and Cander (14) by using a ratio of red-cell membrane permeability to that of its interior of 2.5 , which they found to be the average value. These values for $\theta$ were calculated on the basis of a CO capacity of $20 \mathrm{ml}$ per $100 \mathrm{ml}$ blood. Since each subject served as his own control and no significant change in oxygen capacity in an individual subject was noticed under our experimental conditions (Table I), no corrections were made for variations of $\mathrm{CO}$ capacity from $20 \mathrm{ml}$ per $100 \mathrm{ml}$ blood. It is true that variations in $\theta$, while not altering the direction of change in an individual, could vary the degree of change. Since the $\mathrm{O}_{2}$ capacity for this group was actually $19.2 \pm 0.7$, however, the variability is so small that the statistics would not be altered.

Lung compliance $\left(C_{L}\right) . C_{L}$ was measured by a linear pneumotachograph-integrator system and an esophageal balloon as described by Mead and Whittenberger (15). The expressed values for $C_{L}$ represent the mean of values obtained over six to ten slow tidal volumes. Just before each determination, the subject made full inspirations so that the $C_{L}$ changes that Ferris and Pollard (16) found associated with quiet breathing could be avoided. When $C_{L}$ was determined during $\mathrm{G}$-suit inflation, the subjects breathed into a recording spirometer so placed that they were able to observe their respiratory ex-

TABLE II

Hemodynamic changes after atropine sulfate, $2.0 \mathrm{mg}$ iv, in 13 normal supine subjects

\begin{tabular}{|c|c|c|c|c|c|c|c|c|c|c|}
\hline & \multicolumn{2}{|c|}{ CI* } & \multicolumn{2}{|c|}{ HR } & \multicolumn{2}{|c|}{ SI } & \multicolumn{2}{|c|}{ RAP } & \multicolumn{2}{|c|}{ PAP } \\
\hline & B & A & B & $\overline{\mathrm{A}}$ & $\bar{B}$ & $\bar{A}$ & B & $\bar{A}$ & B & A \\
\hline Mean & 2.96 & 3.64 & 65 & 104 & 47 & 35 & 7 & 4 & 19 & 14 \\
\hline SD & 0.42 & 0.74 & 9 & 11 & 6 & 8 & & 2 & 5 & 4 \\
\hline $\mathrm{p}$ & & 0.01 & \multicolumn{2}{|c|}{$<0.001$} & \multicolumn{2}{|c|}{$<0.001$} & & & \multicolumn{2}{|c|}{0.005} \\
\hline
\end{tabular}

${ }^{*} \mathrm{CI}=$ cardiac index, liters per minute per meter squared; $\mathrm{HR}=$ heart rate, beats per minute; $\mathrm{SI}=$ stroke index, milliliters per meter squared; RAP = mean right atrial pressure, millimeters $\mathrm{Hg} ; \mathrm{PAP}=$ mean pulmonary artery pressure, $\mathrm{mm} \mathrm{Hg}$ (eight subjects); $\mathrm{B}=$ before atropine; and $\mathrm{A}=$ after atropine. 
TABLE III

Hemodynamic effects of $G$-suit inflation before and after atropine, $2.0 \mathrm{mg}$ iv, in eight normal supine subjects

\begin{tabular}{|c|c|c|c|c|}
\hline & \multicolumn{2}{|c|}{ Before atropine } & \multicolumn{2}{|c|}{ After atropine } \\
\hline & Control & Suit inflated & Control & Suit inflated \\
\hline $\begin{array}{l}\text { Cardiac index } \\
L / \min / m^{2}\end{array}$ & $3.12 \pm 0.42$ & $\underset{S}{S} \underset{\rightarrow}{2.95 \pm 2.52}$ & $3.70 \pm 0.76$ & $s .76 \pm 0.45$ \\
\hline $\begin{array}{l}\text { Mean right atrial } \\
\text { pressure, } m m ~ H g\end{array}$ & $7 \pm 2$ & $\underset{\rightarrow}{01}{ }_{\rightarrow}^{15 \pm 5}$ & $4 \pm 2$ & $\underset{\rightarrow}{01} \quad 7 \pm 3$ \\
\hline $\begin{array}{l}\text { Mean pulmonary artery } \\
\text { pressure, } m m ~ H g\end{array}$ & $19 \pm 5$ & $\underset{\rightarrow}{01} 26 \pm 4$ & $14 \pm 5$ & $\underset{\rightarrow}{01} \stackrel{19 \pm 6}{\rightarrow}$ \\
\hline
\end{tabular}

cursions. Each subject practised until he was able to control his breathing to avoid shifts in functional residual capacity (FRC) during suit inflation. $C_{L}$ was measured before and 30 seconds after abrupt inflation of the $\mathrm{G}$ suit to $100 \mathrm{~mm} \mathrm{Hg}$ before and 10 minutes after atropine.

Arterial blood and physiologic dead space [ $\left.V_{D}(p h y s)\right]$ measurements. Arterial oxyhemoglobin capacity and saturation were determined spectrophotometrically (17, 18). Arterial $\mathrm{P}_{\mathrm{CO}_{2}}$ and the $\mathrm{P}_{\mathrm{CO}_{2}}$ of mixed expired gas were determined with a $\mathrm{P}_{\mathrm{CO}_{2}}$ electrode system. ${ }^{3} V_{D}$ (phys) was determined by the method of Riley and co-workers (19) in seated subjects before and 10 minutes after administration of atropine.

Lung volumes and airway resistance. FRC and airway resistance were determined in the total body plethysmograph by the techniques described by DuBois and associates (20) and DuBois, Botelho, and Comroe (21). Ex-

TABLE IV

Change in estimated pulmonary blood volume after atropine, $2 \mathrm{mg}$ iv, in normal supine subjects

\begin{tabular}{ll}
\hline & $\begin{array}{c}\text { Percentage of } \\
\text { change in } \\
\text { estimated } \\
\text { pulmonary } \\
\text { blood volume } \\
\text { after atropine* }\end{array}$ \\
\hline Name & -10.5 \\
J.W. & -1.3 \\
D.W. & -11.2 \\
M.L. & -10.6 \\
B.B. & -9.8 \\
M.P. & -12.0 \\
Mean & -9.2 \\
SD & 3.9 \\
p & 0.005
\end{tabular}

* (cpm after atropine-background cpm)/(cpm before atropine-background cpm) $\times 100$.

${ }^{3}$ Model 103, Instrumentation Laboratories, Boston, Mass. piratory reserve volume was measured on a Collins Stead-Wells spirometer and subtracted from FRC to obtain residual volume for use in calculation of $D_{L}$.

In some subjects, determinations of $\mathrm{FRC}$ and volume of the slowly ventilated component of the FRC $\left(V_{s}\right)$ were also carried out by the open-circuit helium technique of Hickam, Blair, and Frayser (22).

Statistics. The effects of atropine, G-suit inflation, and positive-pressure breathing were examined statistically by the "paired comparison" $t$, with each subject serving as his own control (23).

\section{RESULTS}

Hemodynamic effects of atropine. After atropine, in 13 subjects, cardiac index increased $\left(2.96 \pm 0.42\right.$ to $3.64 \pm 0.74 \mathrm{~L}$ per minute per $\mathrm{m}^{2}$, $\mathrm{p}=0.01)$, heart rate increased $(65 \pm 9$ to $104 \pm$ 11 beats per minute, $p<0.001)$, stroke index decreased ( $47 \pm 6$ to $35 \pm 8 \mathrm{ml}$ per $\mathrm{m}^{2}, \mathrm{p}<0.001$ ), mean right atrial pressure decreased $(7 \pm 2$ to $4 \pm 2 \mathrm{~mm} \mathrm{Hg}, \mathrm{p}<0.001$ ), and mean pulmonary artery pressure decreased ( $19 \pm 5$ to $14 \pm 4 \mathrm{~mm}$ $\mathrm{Hg}, \mathrm{p}=0.005$ ) (Table II).

G-suit inflation did not alter cardiac index either before or after atropine administration (Table III). Before atropine, mean right atrial pressure, measured 30 seconds after suit inflation, was consistently increased $(7 \pm 2$ to $15 \pm 5 \mathrm{~mm} \mathrm{Hg}, \mathrm{p}=$ $0.01)$. After atropine, suit inflation produced a smaller increase in right atrial pressure $(4 \pm 2$ to $7 \pm 3 \mathrm{~mm} \mathrm{Hg}, \mathrm{p}=0.01$ ).

Mean pulmonary artery pressure was increased by suit inflation $(19 \pm 5$ to $26 \pm 4 \mathrm{~mm} \mathrm{Hg}, \mathrm{p}=$ 0.01 ). After atropine, mean pulmonary artery pressure was increased only to the preatropine level $(14 \pm 5$ to $19 \pm 6 \mathrm{~mm} \mathrm{Hg}, \mathrm{p}=0.01)$. 
Pulmonary blood volume. The data of Table IV demonstrate a consistent decrease, averaging $9.2 \pm 3.9 \%$, in the counts detected over the right mid-lung field after atropine in six supine subjects.

$D_{L}$ and $V_{c}$. The data in Tables V and VI demonstrate a decrease in $D_{L}$ after atropine administration in both seated and supine subjects. In the seated subjects, mean $D_{L}$ decreased from $25.2 \pm 3.4$ to $22.0 \pm 3.8 \mathrm{ml} \mathrm{CO}$ per $\mathrm{mm} \mathrm{Hg}$ per minute $(\mathrm{p}=0.05)$. In supine subjects, atropine decreased $D_{L}$ from $31.3 \pm 7.3$ to $25.5 \pm 7.3 \mathrm{ml}$ $\mathrm{CO}$ per $\mathrm{mm} \mathrm{Hg}$ per minute $(\mathrm{p}=0.005)$. In both supine and seated subjects, pulmonary $V_{c}$ was decreased after atropine (seated, $85 \pm 26$ to
$66 \pm 12 \mathrm{ml}, \mathrm{p}=0.01$; supine, $112 \pm 42$ to $93 \pm$ $34 \mathrm{ml}, \mathrm{p}=0.025)$. Atropine had no significant effect on $V_{\boldsymbol{A}}$.

Before atropine, G-suit inflation increased $D_{L}$ $(25.2 \pm 3.4$ to $30.6 \pm 5.3 \mathrm{ml} \mathrm{CO}$ per minute per $\mathrm{mm} \mathrm{Hg}, \mathrm{p}=0.005)$ and $V_{c}(85 \pm 16$ to $117 \pm$ $26 \mathrm{ml}, \mathrm{p}=0.005)$, but caused no statistically significant changes in $V_{A}$ (Table $\mathrm{V}$ ). After atropine, suit inflation increased $D_{L}(22.0 \pm 3.8$ to $25.7 \pm$ $5.3 \mathrm{ml} \mathrm{CO}$ per $\mathrm{mm} \mathrm{Hg}$ per minute, $\mathrm{p}=0.025$ ) and $V_{c}(66 \pm 12$ to $79 \pm 22 \mathrm{ml}, \mathrm{p}=0.05)$ without significant change in $V_{A}$ (Table V). After atropine, then, suit inflation increased both $D_{L}$ and $V_{c}$, but not to the high level attained before atropine.

TABLE V

Pulmonary diffusing capacity $\left(D_{L}\right)$, pulmonary capillary blood volume $\left(V_{c}\right)$, and alveolar volume ( $\left.V_{A}\right)$ in normal seated subjects: the effects of atropine, $2.0 \mathrm{mg}$ iv, and G-suit inflation

\begin{tabular}{|c|c|c|c|c|}
\hline Subject & Condition & $V_{A}$ & $D_{L}$ & $V_{c}$ \\
\hline J.O. & $\begin{array}{l}\text { Seated } \\
\text { G suit } \\
\text { Atropine } \\
\text { G suit }\end{array}$ & $\begin{array}{c}m l \\
4,960 \\
5,390 \\
4,390 \\
4,120\end{array}$ & $\begin{array}{c}\mathrm{ml} / \mathrm{min} / \mathrm{mm} \mathrm{Hg} \\
24.4 \\
32.4 \\
21.3 \\
26.9\end{array}$ & $\begin{array}{r}m l \\
100 \\
125 \\
83 \\
115\end{array}$ \\
\hline B.S. & $\begin{array}{l}\text { Seated } \\
\text { G suit } \\
\text { Atropine } \\
\text { G suit }\end{array}$ & $\begin{array}{l}3,890 \\
3,520 \\
3,700 \\
3,560\end{array}$ & $\begin{array}{l}20.2 \\
22.8 \\
20.1 \\
19.6\end{array}$ & $\begin{array}{l}61 \\
70 \\
52 \\
46\end{array}$ \\
\hline D.F. & $\begin{array}{l}\text { Seated } \\
\text { G suit } \\
\text { Atropine } \\
\text { G suit }\end{array}$ & $\begin{array}{l}4,910 \\
4,530 \\
4,650 \\
4,590\end{array}$ & $\begin{array}{l}26.9 \\
30.0 \\
18.2 \\
21.6\end{array}$ & $\begin{array}{r}84 \\
125 \\
67 \\
80\end{array}$ \\
\hline D.M. & $\begin{array}{l}\text { Seated } \\
\text { G suit } \\
\text { Atropine } \\
\text { G suit }\end{array}$ & $\begin{array}{l}4,500 \\
4,340 \\
4,370 \\
4,400\end{array}$ & $\begin{array}{l}25.8 \\
30.9 \\
25.4 \\
29.2\end{array}$ & $\begin{array}{r}95 \\
111 \\
55 \\
67\end{array}$ \\
\hline H.H. & $\begin{array}{c}\text { Seated } \\
\text { G suit } \\
\text { Atropine } \\
\text { G suit }\end{array}$ & $\begin{array}{l}5,450 \\
5,020 \\
5,260 \\
5,350\end{array}$ & $\begin{array}{l}22.5 \\
26.8 \\
19.1 \\
21.9\end{array}$ & $\begin{array}{r}91 \\
111 \\
64 \\
69\end{array}$ \\
\hline J.M. & $\begin{array}{l}\text { Seated } \\
\text { G suit } \\
\text { Atropine } \\
\text { G suit }\end{array}$ & $\begin{array}{l}4,760 \\
5,000 \\
5,450 \\
5,360\end{array}$ & $\begin{array}{l}31.0 \\
39.9 \\
28.9 \\
37.4\end{array}$ & $\begin{array}{r}100 \\
159 \\
80 \\
91\end{array}$ \\
\hline J.W. & $\begin{array}{l}\text { Seated } \\
\text { G suit } \\
\text { Atropine } \\
\text { G suit }\end{array}$ & $\begin{array}{l}3,860 \\
3,480 \\
3,880 \\
3,475\end{array}$ & $\begin{array}{l}26.0 \\
31.1 \\
20.9 \\
22.0\end{array}$ & $\begin{array}{r}64 \\
118 \\
60 \\
83\end{array}$ \\
\hline Mean $\pm \mathrm{SD}$ & $\begin{array}{l}\text { Seated } \\
\text { G suit } \\
\text { Atropine } \\
\text { G suit }\end{array}$ & $\begin{array}{l}4,618 \pm 582 \\
4,468 \pm 746 \\
4,528 \pm 652 \\
4,407 \pm 763\end{array}$ & $\begin{array}{l}25.2 \pm 3.4 \\
30.6 \pm 5.3 \\
22.0 \pm 3.8 \\
25.7 \pm 5.3\end{array}$ & $\begin{array}{r}85 \pm 16 \\
117 \pm 26 \\
66 \pm 12 \\
79 \pm 22\end{array}$ \\
\hline $\mathrm{p}$ & $\begin{array}{l}\text { Seated-atropine } \\
\text { Seated-G suit } \\
\text { Atropine-G suit } \\
\text { and atropine }\end{array}$ & $\begin{array}{l}\text { NS } \\
\text { NS } \\
\text { NS }\end{array}$ & $\begin{array}{l}0.05 \\
0.005 \\
0.025\end{array}$ & $\begin{array}{l}0.01 \\
0.005 \\
0.05\end{array}$ \\
\hline
\end{tabular}


TABLE VI

Pulmonary diff using capacity $\left(D_{L}\right)$, pulmonary capillary blood volume $\left(V_{c}\right)$, and alveolar volume $\left(V_{A}\right)$ in normal supine subjects: the effects of atropine, $2.0 \mathrm{mg}$ iv, and positive-pressure breathing, $10 \mathrm{~mm} \mathrm{Hg}$

\begin{tabular}{|c|c|c|c|c|}
\hline Subject & Condition & $V_{A}$ & $D_{L}$ & $V_{c}$ \\
\hline J.O. & $\begin{array}{l}\text { Supine } \\
\text { Positive pressure } \\
\text { Atropine } \\
\text { Positive pressure }\end{array}$ & $\begin{array}{c}m l \\
4,760 \\
4,720 \\
4,650 \\
4,680\end{array}$ & $\begin{array}{c}\mathrm{ml} / \mathrm{min} / \mathrm{mm} \mathrm{Hg} \\
23.1 \\
24.1 \\
19.1 \\
19.9\end{array}$ & $\begin{array}{r}m l \\
182 \\
93 \\
125 \\
39\end{array}$ \\
\hline B.S. & $\begin{array}{l}\text { Supine } \\
\text { Positive pressure } \\
\text { Atropine } \\
\text { Positive pressure }\end{array}$ & $\begin{array}{l}4,510 \\
4,200 \\
4,200 \\
3,990\end{array}$ & $\begin{array}{l}27.1 \\
22.5 \\
17.8 \\
10.3\end{array}$ & $\begin{array}{l}56 \\
27 \\
44\end{array}$ \\
\hline D.F. & $\begin{array}{l}\text { Supine } \\
\text { Positive pressure } \\
\text { Atropine } \\
\text { Positive pressure }\end{array}$ & $\begin{array}{l}6,080 \\
5,450 \\
4,820 \\
5,290\end{array}$ & $\begin{array}{l}32.8 \\
23.4 \\
19.3 \\
22.3\end{array}$ & $\begin{array}{l}72 \\
93 \\
76 \\
47\end{array}$ \\
\hline D.M. & $\begin{array}{l}\text { Supine } \\
\text { Positive pressure } \\
\text { Atropine } \\
\text { Positive pressure }\end{array}$ & $\begin{array}{l}5,140 \\
4,900 \\
5,340 \\
5,530\end{array}$ & $\begin{array}{l}29.6 \\
28.0 \\
28.4 \\
28.8\end{array}$ & $\begin{array}{r}125 \\
117 \\
105 \\
95\end{array}$ \\
\hline H.H. & $\begin{array}{l}\text { Supine } \\
\text { Positive pressure } \\
\text { Atropine } \\
\text { Positive pressure }\end{array}$ & $\begin{array}{l}4,780 \\
4,810 \\
5,120 \\
4,810\end{array}$ & $\begin{array}{l}26.2 \\
19.4 \\
22.9 \\
19.3\end{array}$ & $\begin{array}{r}100 \\
63 \\
80 \\
100\end{array}$ \\
\hline J.M. & $\begin{array}{l}\text { Supine } \\
\text { Positive pressure } \\
\text { Atropine } \\
\text { Positive pressure }\end{array}$ & $\begin{array}{l}6,780 \\
6,910 \\
7,180 \\
7,150\end{array}$ & $\begin{array}{l}45.3 \\
42.6 \\
38.4 \\
41.3\end{array}$ & $\begin{array}{l}154 \\
125 \\
154 \\
117\end{array}$ \\
\hline J.W. & $\begin{array}{l}\text { Supine } \\
\text { Positive pressure } \\
\text { Atropine } \\
\text { Positive pressure }\end{array}$ & $\begin{array}{l}3,840 \\
4,050 \\
3,980 \\
3,480\end{array}$ & $\begin{array}{l}27.8 \\
27.6 \\
24.7 \\
21.9\end{array}$ & $\begin{array}{l}91 \\
83 \\
70 \\
71\end{array}$ \\
\hline B.Mo. & $\begin{array}{l}\text { Supine } \\
\text { Positive pressure } \\
\text { Atropine } \\
\text { Positive pressure }\end{array}$ & $\begin{array}{l}7,180 \\
7,260 \\
7,680 \\
7,490\end{array}$ & $\begin{array}{l}38.8 \\
35.6 \\
33.7 \\
29.4\end{array}$ & $\begin{array}{r}118 \\
100 \\
88 \\
67\end{array}$ \\
\hline Mean $\pm S D$ & $\begin{array}{l}\text { Supine } \\
\text { Positive pressure } \\
\text { Atropine } \\
\text { Positive pressure }\end{array}$ & $\begin{array}{l}5,380 \pm 1,170 \\
5,290 \pm 1,195 \\
5,370 \pm 1,350 \\
5,350 \pm 1,350\end{array}$ & $\begin{array}{l}31.3 \pm 7.3 \\
27.9 \pm 7.7 \\
25.5 \pm 7.3 \\
24.1 \pm 9.1\end{array}$ & $\begin{array}{r}112 \pm 42 \\
88 \pm 31 \\
93 \pm 34 \\
76 \pm 29\end{array}$ \\
\hline $\mathrm{p}$ & $\begin{array}{l}\text { Supine-atropine } \\
\text { Supine-positive pressure } \\
\text { Atropine-positive } \\
\quad \text { pressure and atropine }\end{array}$ & $\begin{array}{l}\text { NS } \\
\text { NS } \\
\text { NS }\end{array}$ & $\begin{array}{c}0.005 \\
0.025 \\
\text { NS }\end{array}$ & $\begin{array}{c}0.025 \\
0.025 \\
\text { NS }\end{array}$ \\
\hline
\end{tabular}

Positive-pressure breathing (Table VI) decreased $D_{L}(31.3 \pm 7.3$ to $27.9 \pm 7.7 \mathrm{ml} \mathrm{CO}$ per $\mathrm{mm} \mathrm{Hg}$ per minute, $\mathrm{p}=0.025)$. $D_{L}$ during positive-pressure breathing $(27.9 \pm 7.7 \mathrm{ml} \mathrm{CO}$ per $\mathrm{mm} \mathrm{Hg}$ per minute) was not statistically different from $D_{L}$ after atropine $(25.5 \pm 7.3 \mathrm{ml} \mathrm{CO}$ per $\mathrm{mm}$ $\mathrm{Hg}$ per minute, $\mathrm{p}=0.1) . \quad V_{c}$ after atropine (93 \pm $34 \mathrm{ml}$ ) was not statistically different from $V_{c}$ during positive-pressure breathing before atropine $(88 \pm 31 \mathrm{ml})$.
After atropine, the changes observed in $D_{L}$ and $V_{c}$ during positive-pressure breathing were not statistically significant. $V_{A}$ was not affected by positive-pressure breathing either before or after atropine.

$C_{L}$. $C_{L}$ was decreased by G-suit inflation $(0.193$ \pm 0.026 to $0.137 \pm 0.046 \mathrm{~L}$ per $\mathrm{cm} \mathrm{H}_{2} \mathrm{O}, \mathrm{p}=$ 0.005) (Table VII). Atropine increased $C_{L}$ $\left(0.193 \pm 0.026\right.$ to $0.234 \pm 0.047 \mathrm{~L}$ per $\mathrm{cm} \mathrm{H}_{2} \mathrm{O}$, $\mathrm{p}=0.025)$. After atropine, suit inflation de- 
creased $C_{L}(0.234 \pm 0.047$ to $0.201 \pm 0.054, \mathrm{p}=$ $0.05)$, but not to the low level obtained during suit inflation before atropine.

Lung volumes, airway resistance, and blood oxygen. Atropine produced no change in FRC, "slow space," $\mathrm{O}_{2}$ capacity, or oxyhemoglobin saturation. Airway resistance was decreased slightly after atropine $(1.82$ to $1.45 \mathrm{~L}$ per second per $\mathrm{cm} \mathrm{H}_{2} \mathrm{O}, \mathrm{p}=0.05$ ), and $V_{\left.D_{(\text {phys }}\right)}$ was increased after atropine $(139 \pm 13$ to $166 \pm 24 \mathrm{ml}$, $\mathrm{p}=0.01)($ Table I).

Summary of results. Table VIII summarizes the effects of atropine, G-suit inflation, and positive-pressure breathing.

\section{DISCUSSION}

In the present study, the iv administration of atropine sulfate not only increased heart rate and
TABLE VII

Pulmonary compliance $\left(C_{L}\right)$ in normal seated subjects: the effects of atropine, $2.0 \mathrm{mg}$ iv, and $\mathrm{G}$-suit inflation

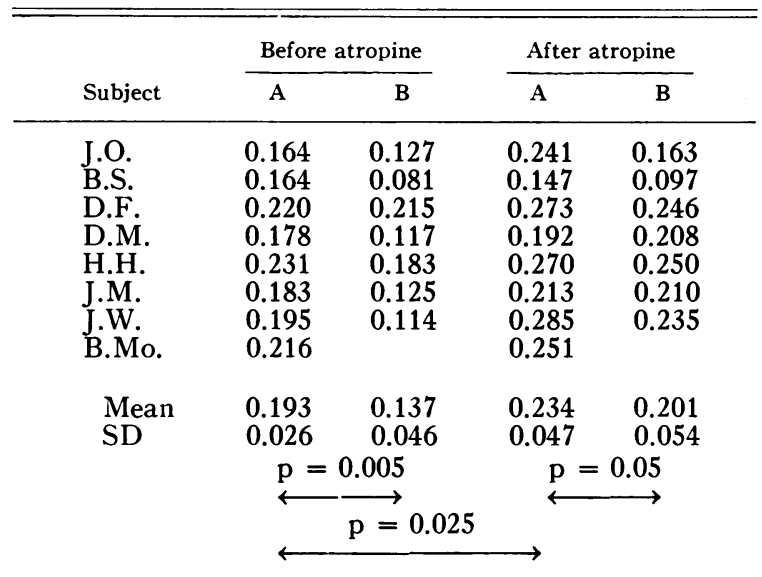

* $\mathrm{A}=$ before $\mathrm{G}$-suit inflation; $\mathrm{B}=30$ seconds after G-suit inflation to $100 \mathrm{~mm} \mathrm{Hg}$.

TABLE VIII

Summary of the effects observed ${ }^{*}$

\begin{tabular}{|c|c|c|c|c|c|}
\hline & Atropine & G suit & $\begin{array}{l}\text { Positive- } \\
\text { pressure } \\
\text { breathing }\end{array}$ & $\begin{array}{l}\text { Atropine } \\
+G \text { suit }\end{array}$ & $\begin{array}{c}\text { Atropine } \\
\text { + positive- } \\
\text { pressure } \\
\text { breathing } \delta\end{array}$ \\
\hline Cardiac index & $\uparrow \uparrow$ & $\mathrm{NC} \ddagger$ & & $\mathrm{NC}$ & \\
\hline $\begin{array}{l}\text { Right atrial mean } \\
\text { pressure }\end{array}$ & $\downarrow$ & $\uparrow$ & & $\uparrow$ & \\
\hline $\begin{array}{l}\text { Pulmonary artery } \\
\text { mean pressure }\end{array}$ & $\downarrow$ & $\uparrow$ & & $\uparrow$ & \\
\hline $\begin{array}{l}\text { Pulmonary blood } \\
\text { volume }\end{array}$ & $\downarrow$ & & & & \\
\hline Lung compliance & $\uparrow$ & $\downarrow$ & & $\downarrow$ & \\
\hline $\begin{array}{l}\text { Pulmonary diffusing } \\
\text { capacity }\end{array}$ & $\downarrow$ & $\uparrow$ & $\downarrow$ & $\uparrow$ & $\mathrm{NC}$ \\
\hline $\begin{array}{l}\text { Pulmonary capillary } \\
\text { blood volume }\end{array}$ & $\downarrow$ & $\uparrow$ & $\downarrow$ & $\uparrow$ & $\mathrm{NC}$ \\
\hline $\begin{array}{l}\text { Functional residual } \\
\text { capacity }\end{array}$ & $\mathrm{NC}$ & & & & \\
\hline Airway resistance & $\downarrow$ & & & & \\
\hline $\begin{array}{l}\text { Slowly ventilated } \\
\text { space }\end{array}$ & $\mathrm{NC}$ & & & & \\
\hline $\begin{array}{l}\text { Physiologic dead } \\
\text { space }\end{array}$ & $\uparrow$ & & & & \\
\hline $\begin{array}{l}\text { Arterial oxyhemo- } \\
\text { globin saturation }\end{array}$ & $\mathrm{NC}$ & & & & \\
\hline $\begin{array}{l}\text { Arterial oxygen } \\
\text { capacity }\end{array}$ & $\mathrm{NC}$ & & & & \\
\hline
\end{tabular}

* Conditions are as defined in the section on methods.

$\dagger$ Arrows indicate the direction of statistically significant change $(p=0.05)$.

NC, no change.

$\$$ Compared with atropine alone. 
cardiac index and decreased right atrial pressure and stroke volume, as others have reported $(1,2)$, but also caused a decrease in pulmonary artery pressure. Previous direct measurements of intrapleural pressure have suggested that atropine probably does not produce changes in intrathoracic pressure (5), and therefore, the observed changes in central vascular pressures should reasonably reflect changes in transmural pressures. In the absence of simultaneous left atrial pressure measurements, consideration of the effects of atropine on pulmonary vascular resistance is speculative, yet, given the combination of increased cardiac output and decreased pulmonary arterial pressure, it can be argued that either pulmonary vascular resistance must have decreased or left atrial pressure decreased considerably more than pulmonary arterial pressure or right atrial pressure.

Acute central vascular engorgement produced by G-suit inflation to $100 \mathrm{~mm} \mathrm{Hg}$ did not increase cardiac index, measured by dye injection $30 \mathrm{sec}-$ onds after suit inflation, either before or after atropine. Ross, Frayser, and Hickam (24) previously reported no increase in cardiac output with G-suit inflation. Since all these subjects were supine when studied, the data are not at variance with those of Weissler, Leonard, and Warren (25), who found an increase in cardiac index during G-suit inflation in tilted subjects after atropine. The absence of an increase in cardiac index as a result of an increase in right atrial pressure produced by G-suit inflation for 30 seconds is consistent with the observations of others $(26,27)$ that acute elevation of previously normal right atrial and pulmonary vascular pressures by iv infusion does not consistently increase cardiac output in normal subjects. A transient increase in cardiac output during the first 30 seconds after $\mathrm{G}$-suit inflation is not excluded.

In the present study, G-suit inflation caused a mean increase in central venous pressure of $8 \mathrm{~mm}$ $\mathrm{Hg}$ in eight supine subjects. Previous observations in similar subjects have shown that intrapleural pressure rises only an average of $1.35 \mathrm{~mm}$ $\mathrm{Hg}$ during $\mathrm{G}$-suit inflation (5). After atropine, however, the increase in right atrial pressure produced by G-suit inflation was diminished. Two alternative explanations are suggested for the decrease in central vascular pressure after atropine and for the difference in the effect of G-suit infla- tion: a) after atropine, blood shifts out of the central vascular reservoir into an area that is not effectively compressed by the G suit, or $b$ ) after atropine, the compliance of the pulmonary vascular reservoir is increased so that blood is contained at a lower pressure, and the blood transferred to the lungs by G-suit inflation is accommodated at a lower pressure.

Our observations with the external counting technique for estimation of change in pulmonary blood volume (Table IV) indicate that there is less blood in the lungs of supine subjects after atropine administration and support the concept that the shift of blood is away from the lung. $D_{L}$ and $V_{c}$, whether measured in seated or supine subjects, were also decreased after atropine administration. Acute central vascular engorgement produced by G-suit inflation moves blood into the lungs (7) and increases $D_{L}(4)$, again demonstrated in the present study. In these subjects, G-suit inflation after atropine increased central vascular pressures only to about the levels present before atropine was given and caused increases in $D_{L}$ and $V_{c}$ to much lower levels than those that were attained during G-suit inflation before atropine.

Fenn, Otis, Rahn, Chadwick, and Hegnauer (28) have shown that there is a shift of blood out of the thorax during positive-pressure breathing. Positive-pressure breathing at $10 \mathrm{~mm} \mathrm{Hg}$ in normal supine men does not affect cardiac output (5). This does not imply that greater amounts of positive pressure would not decrease cardiac output (29). In the present study, this procedure was associated with decreases in $D_{L}$ and $V_{c}$. After atropine, $D_{L}$ and $V_{c}$ were not further decreased by positive-pressure breathing. These decreases in $D_{L}$ and $V_{c}$ during $10 \mathrm{~mm} \mathrm{Hg}$ positivepressure breathing are not unlike changes previously observed during Valsalva maneuvers (4, 10).

The absence of changes in FRC, slowly ventilated space $\left(V_{S}\right)$, and arterial oxyhemoglobin saturation along with the decreased airway resistance after atropine provide evidence that the effects of atropine on $D_{L}$ are not the result of restricted distribution of gas inspired for the $D_{L}$ measurement.

These observations of the effects of atropine on $D_{L}$ and $V_{c}$ have certain implications concerning 
over-all pulmonary capillary function. In 1915, Krogh (12) observed an increase in $D_{L}$ during exercise. The increase during exercise has not been explained (24). It is known that hyperventilation does not increase breath-holding $D_{L}$ (24) and that neither breath-holding nor steadystate $D_{L}$ are increased by acute increases in pulmonary blood flow in man $(24,30)$. In fact, as was pointed out by Hatch (31), consideration of the CO capacity of blood and the rate of $\mathrm{CO}$ diffusion suggests that increased pulmonary blood flow should not affect $D_{L_{\mathrm{co}}}$ at low alveolar CO tensions, unless there is an associated increase in the volume of blood actually involved in gas exchange at any instant in time. In other words, for an increased pulmonary blood flow to increase $D_{L_{\mathrm{co}}}$, either the area for diffusion, or the volume of blood into which gas is diffusing at any instant must increase, and these two are geometrically related factors. Observations thus far available demonstrate only two naturally occurring situations of increased blood flow that are associated with increased $V_{c}$ and $D_{L}$ : the increased flow associated with atrial septal defect (32) and the increased flow with exercise. It is not understood how either increases the instantaneous volume of capillary blood available for diffusion. In man, two other situations have been shown to increase $D_{L}$ acutely, and these alter pulmonary vascular pressure and volume, but produce little if any change in cardiac output. Ross, Lord, and Ley (4) found an abrupt increase in $D_{L}$ during acute central vascular engorgement, and Lewis, Lin, Noe, and Komisaruk (33) found an increase in $D_{L}$ when subjects change from the erect to the supine position. Lewis, McElroy, Hayford-Welsing, and Samberg (34) also found a decrease in $D_{L}$ during infusion of trimethapan (Arfonad), a procedure designed to decrease pulmonary vascular transmural pressure. Our observations on the effects of atropine in man are in agreement with those of Rosenberg and Forster (35), who, working with isolated cat lungs, concluded that $D_{L}$ is primarily dependent upon pulmonary vascular transmural pressure. No independent effect of flow was observed.

These observations with atropine tend to separate the factors of pressure and flow as determinants of $D_{L}$ in man and suggest that $D_{L}$ is more dependent on pulmonary transmural pressure.
This does not imply that pulmonary vascular pressure is the sole hemodynamic determinant of acute changes in $D_{L}$. The instantaneous pulmonary capillary blood volume certainly increases with moderate exercise, whereas in normal man there is little or no increase in pulmonary arterial pressure with moderate exercise $(36,37)$. Such factors as the evenness of distribution of perfusion with regard to ventilation, the compliance of the pulmonary vascular bed, and the effect of systemic venous activity may be of prime importance, but are as yet unresolved problems.

It is uncertain from the present data whether the events occurring in the pulmonary vascular bed after atropine administration are specific direct effects of atropine, or reflect pressure responses to actions of atropine elsewhere. The observed association of decreased $D_{L}, V_{c}$, and pulmonary blood volume with decreased pulmonary arterial pressure and increased cardiac output suggests that the changes in the pulmonary vascular bed are in response to pooling of blood elsewhere.

Severinghaus and Stupfel (38) found an increase in anatomical dead space in man after atropine and interpreted this change as evidence of bronchomotor tone and an effect of atropine on this tone. Our finding of decreased airway resistance after atropine is in general agreement with this concept. The observed increase in $V_{D_{\text {(phys) }}}$ could reflect an enlargement of anatomic airway volume. The possibility of increased alveolar dead space associated with uneven distribution of perfusion and ventilation after atropine is not excluded, although the small increase in $V_{D_{\text {(phys) }} \text { suggests that total closure of pulmonary }}$ capillaries must not contribute greatly to thè decreased $D_{L}$ and $V_{c}$ observed. This implies a more evenly distributed decrease in pulmonary capillary volume without actual capillary closure.

The dependence in man of dynamic $C_{L}$ upon pulmonary vascular pressure, or volume, or both, was shown by Bondurant and co-workers $(7,39$, 40 ), who produced acute central vascular engorgement by G-suit inflation and decreased $C_{L}$ by this maneuver. The effects of atropine on $C_{L}$ suggest that, just as increased pulmonary vascular pressures decrease $C_{L}$, decreased pulmonary vascular pressures and volume increase $C_{L}$. 
The observed association of an increase in dynamic $C_{L}$ and a decrease in airway resistance after atropine suggests that the change in $C_{L}$ might be the consequence of more even distribution of the inspired air, but the absence of change in the slowly ventilated component of the FRC after atropine suggests that the change in $C_{L}$ is not caused by altered distribution of ventilation. Furthermore, a change in $C_{L}$ caused by decreased airway resistance would suggest that $C_{L}$, in normal men, is affected by breathing frequency and that $C_{L}$ should increase at decreased breathing frequencies; however, Otis and co-workers (41) found that $C_{L}$ is not frequency dependent in normal men. It would seem, therefore, that the observed increase in $C_{L}$ after atropine is not the result of the decreased airway resistance. Since atropine produced no change in FRC, the change in $C_{L}$ after atropine cannot be attributed to a change in lung volume.

The possibility cannot be denied that the observed change in $C_{L}$ after atropine is not real, but is the result of a change in esophageal compliance. If atropine increased the rigidity of the esophagus, similar changes in $C_{L}$ measured by this method would be observed. The information available concerning the effects of atropine on alimentary smooth muscle, however, suggests that the esophagus should become more relaxed after atropine (42). Consequently, subject to the inherent reservations associated with the use of the esophagus as a site for pressure measurement in respiratory mechanics, it would seem that the observed increase in $C_{L}$ after atropine is dependent upon the decreased pulmonary vascular pressure and volume after atropine.

These observations of the effects of atropine on $C_{L}, D_{L}, V_{c}$, and pulmonary blood volume seem to substantiate the thought that atropine, despite its associated increase in cardiac output, reduces the actual volume of blood in the lung and certainly reduces the volume available for gas exchange. These observations do not define the site to which blood has been translocated after atropine, nor the mechanisms involved. The plethysmographic observations of Horsley and Eckstein (3), who found an increased extremity venomotor tone and decreased extremity venous volume after atropine, suggest that blood is also shifted away from the extremities, so by exclusion, a shift to the splanchnic region seems likely.

It is tempting to consider this effect of atropine the result of its parasympatholytic action. There is evidence, however, that the effects of atropine are more complex and that atropine may, in large doses, have actions similar to sympathetic ganglionic blocking agents (43-45).

\section{SUMMARY}

In normal supine subjects, atropine increases cardiac index and decreases right atrial pressure and pulmonary artery pressure. Changes in pulmonary blood volume, estimated by an external $\mathrm{I}^{131}$-counting technique, suggest that atropine causes a redistribution of the blood volume away from the lung.

With the decrease in pulmonary vascular pressures and volume, pulmonary diffusing capacity $\left(D_{L}\right)$ and pulmonary capillary volume $\left(V_{c}\right)$ decrease, whereas lung compliance $\left(C_{L}\right)$ increases. These changes are not the result of altered distribution of ventilation or changes in lung volume.

Acute pulmonary vascular engorgement produced by G-suit inflation increases right atrial pressure, pulmonary artery pressure, $D_{L}$, and $V_{0}$ and decreases $C_{L}$. Similar, though smaller, changes are produced by G-suit inflation after atropine.

Continuous positive-pressure breathing, a procedure known to move blood out of the chest, decreases $D_{L}$ and $V_{c}$.

These findings suggest that atropine causes a shift of blood out of the lungs into an area where it is not effectively mobilized by G-suit inflation. A specific effect of atropine on the pulmonary vasculature cannot be excluded.

This study demonstrates, in man, the dependence of $D_{L}$ and $V_{c}$ on pulmonary vascular pressures, or volume, or both, and their relative independence of cardiac output.

The decrease in $C_{L}$ after atropine is independent of change in lung volume or distribution of ventilation and probably is a further manifestation of the dependence of $C_{L}$ on pulmonary vascular pressure.

\section{REFERENCES}

1. Berry, J. N., H. K. Thompson, Jr., D. E. Miller, and H. D. McIntosh. Changes in cardiac output, stroke 
volume, and central venous pressure induced by atropine in man. Amer. Heart J. 1959, 58, 204.

2. Gorten, R., J. C. Gunnels, A. M. Weissler, and E. A. Stead, Jr. Effects of atropine and isoproterenol on cardiac output; central venous pressure, and mean transit time of indicators placed at three different sites in the venous system. Circulat. Res. 1961, 9, 979.

3. Horsley, A. W., and J. W. Eckstein. Pressurevolume changes in human forearm veins after atropine administration. Proc. Soc. exp. Biol. (N. Y.) 1959, 100, 822.

4. Ross, J. C., T. H. Lord, and G. D. Ley. Effect of pressure-suit inflation on pulmonary-diffusing capacity. J. appl. Physiol. 1960, 15, 843.

5. Daly, W. J., and R. H. Behnke. The behavior of the venous reservoir as affected by atropine. Trans. Ass. Amer. Phycns 1962, 75, 277.

6. Kinsman, J. M., J. W. Moore, and W. F. Hamilton. Studies on the circulation. I. Injection method: physical and mathematical considerations. Amer. J. Physiol. 1929, 89, 322.

7. Bondurant, S., J. B. Hickam, and J. K. Isley. Pulmonary circulatory effects of acute pulmonary vascular engorgement in normal subjects. J. clin. Invest. 1957, 36, 59.

8. Weissler, A. M., B. H. McCraw, and J. V. Warren. Pulmonary blood volume determined by a radioactive tracer technique. J. appl. Physiol. 1959, 14, 531.

9. Forster, R. E., W. S. Fowler, D. V. Bates, and B. Van Lingen. The absorption of carbon monoxide by the lungs during breathholding. J. clin. Invest. 1954, 33, 1135.

10. Ogilvie, C. M., R. E. Forster, W. S. Blakemore, and J. W. Morton. A standardized breath holding technique for the clinical measurement of the diffusing capacity of the lung for carbon monoxide. J. clin. Invest. 1957, 36, 1.

11. Roughton, F. J. W., and R. E. Forster. Relative importance of diffusion and chemical reaction rates in determining rate of exchange of gases in the human lung, with special reference to true diffusing capacity of pulmonary membrane and volume of blood in the lung capillaries. J. appl. Physiol. 1957, 11, 290

12. Krogh, M. The diffusion of gases through the lungs of man. J. Physiol. (Lond.) 1915, 49, 271.

13. McNeill, R. S., J. Rankin, and R. E. Forster. The diffusing capacity of the pulmonary membrane and the pulmonary capillary blood volume in cardiopulmonary disease. Clin. Sci. 1958, 17, 465.

14. Roughton, F. J. W., R. E. Forster, and L. Cander. Rate at which carbon monoxide replaces oxygen from combination with human hemoglobin in solution and in the red cell. J. appl. Physiol. 1957, 11, 269.

15. Mead, J., and J. L. Whittenberger. Physical properties of human lungs measured during spontaneous respiration. J. appl. Physiol. 1953, 5, 779.
16. Ferris, B. G., Jr., and D. S. Pollard. Effect of deep and quiet breathing on pulmonary compliance in man. J. clin. Invest. 1960, 39, 143.

17. Drabkin, D. L., and J. H. Austin. Spectrophotometric studies. II. Preparations from washed blood cells; nitric oxide hemoglobin and sulfhemoglobin. J. biol. Chem. 1935, 112, 51.

18. Hickam, J. B., and R. Frayser. Spectrophotometric determination of blood oxygen. J. biol. Chem. 1949, 180, 457.

19. Riley, R. L., S. Permutt, S. Said, M. Godfrey, T. O. Cheng, J. B. L. Howell, and R. H. Shepard. Effect of posture on pulmonary dead space in man. J. appl. Physiol. 1959, 14, 339.

20. DuBois, A. B., S. Y. Botelho, G. N. Bedell, R. Marshall, and J. H. Comroe, Jr. A rapid plethysmographic method for measuring thoracic gas volume: a comparison with nitrogen washout method for measuring functional residual capacity in normal subjects. J. clin. Invest. 1956, 35, 322.

21. DuBois, A. B., S. J. Botelho, and J. H. Comroe, Jr. A new method for measuring airway resistance in man using a body plethysmograph: values in normal subjects and in patients with respiratory disease. J. clin. Invest. 1956, 35, 327.

22. Hickam, J. B., E. Blair, and R. Frayser. An opencircuit helium method for measuring functional residual capacity and defective intrapulmonary gas mixing. J. clin. Invest. 1954, 33, 1277.

23. Snedecor, G. W., and W. G. Cochran. Statistical Methods Applied to Experiments in Agriculture and Biology, 5th ed. Ames, Iowa, The Iowa State College Press, 1956.

24. Ross, J. C., R. Frayser, and J. B. Hickam. A study of the mechanism by which exercise increases the pulmonary diffusing capacity for carbon monoxide. J. clin. Invest. 1959, 38, 916.

25. Weissler, A. M., J. J. Leonard, and J. V. Warren. Effects of posture and atropine on the cardiac output. J. clin. Invest. 1957, 36, 1656.

26. Warren, J. V., E. S. Brannon, H. S. Weens, and E. A. Stead, Jr. Effects of increasing the blood volume and right atrial pressure on the circulation of normal subjects by intravenous infusions. Amer. J. Med. 1948, 4, 193.

27. Doyle, J. T., J. S. Wilson, E. H. Estes, and J. H. Warren. The effect of intravenous infusions of physiologic saline solution on the pulmonary arterial and pulmonary capillary pressure in man. J. clin. Invest. 1951, 30, 345 .

28. Fenn, W. O., A. B. Otis, H. Rahn, L. E. Chadwick, and A. H. Hegnauer. Displacement of blood from the lungs by pressure breathing. Amer. J. Physiol. 1947, 151, 258.

29. Werkö, L. The influence of positive pressure breathing on the circulation in man. Acta med. scand. 1947, suppl. 193.

30. Turino, G. M., M. Brandfonbrener, and A. P. Fishman. The effect of changes in ventilation and 
pulmonary blood flow on the diffusing capacity of the lung. J. clin. Invest. 1959, 38, 1186.

31. Hatch, T. F. Carbon monoxide uptake in relation to pulmonary performance. Arch. industr. Hyg. 1952, 6, 1.

32. Bedell, G. N. Comparison of pulmonary diffusing capacity in normal subjects and in patients with intracardiac septal defects. J. Lab. clin. Med. 1961, 57, 269.

33. Lewis, B. M., T-H. Lin, F. E. Noe, and R. Komisaruk. The measurement of pulmonary capillary volume and pulmonary membrane diffusing capacity in normal subjects; the effects of exercise and position. J. clin. Invest. 1958, 37, 1061.

34. Lewis, B. M., W. T. McElroy, E. J. Hayford-Welsing, and L. C. Samberg. The effects of body position, ganglionic blockade and norepinephrine on the pulmonary capillary bed. J. clin. Invest. 1960, 39, 1345.

35. Rosenberg, E., and R. E. Forster. Changes in diffusing capacity of isolated cat lungs with blood pressure and flow. J. appl. Physiol. 1960, 15, 883.

36. Hickam, J. B., and W. H. Cargill. Effect of exercise on cardiac output and pulmonary arterial pressure in normal persons and in patients with cardiovascular disease and pulmonary emphysema. J. clin. Invest. 1948, 27, 10.

37. Riley, R. L., A. Himmelstein, H. L. Motley, H. M. Weiner, and A. Cournand. Studies of the pulmonary circulation at rest and during exercise in normal individuals and in patients with chronic pulmonary disease. Amer. J. Physiol. 1948, 152, 372.

38. Severinghaus, J. W., and M. Stupfel. Respiratory dead space increase following atropine in man, and atropine, vagal or ganglionic blockade and hypothermia in dogs. J. appl. Physiol. 1955, 8, 1055.

39. Bondurant, S., J. Mead, and C. D. Cook. A reevaluation of effects of acute central congestion on pulmonary compliance in normal subjects. J. appl. Physiol. 1960, 15, 875.

40. Daly, W. J., and S. Bondurant. Direct measurement of respiratory pleural pressure changes in normal man. J. appl. Physiol. In press.

41. Otis, A. B., C. B. McKerrow, R. A. Bartlett, J. Mead, M. B. McIlroy, N. J. Selverstone, and E. P. Radford, Jr. Mechanical factors in distribution of pulmonary ventilation. J. appl. Physiol. 1956, 8, 427.

42. Goodman, L. S., and A. Gilman. The Pharmacological Basis of Therapeutics, 2nd ed. New York, Macmillan, 1955, p. 549.

43. Marrazzi, A. Electrical studies on the pharmacology of autonomic synapses. I. The action of parasympathomimetic drugs on sympathetic ganglia. J. Pharmacol. exp. Ther. 1939, 65, 18.

44. Cahen, R. L., and K. M. Tvede. Action of atropine on sympathetic ganglia. Arch. int. Pharmacodyn. 1953, 94, 248.

45. Labate, J. S., and D. Sheehan. Effect of atropine on uterine responses to hypogastric nerve stimulation. Action at the ganglionic synapse. Amer. J. Physiol. 1943, 139, 178. 